\title{
IMMUNOTHERAPY
}

\section{Adoptive cell therapy simplified}

Treatment of patients with metastatic melanoma using tumour-infiltrating lymphocytes (TILs) has, in some cases, resulted in durable remissions of up to 9 years. In a cancer where other targeted therapies have produced impressivebut short-responses, this result is encouraging. However, the generation of the autologous selected TILs that were used in the trials involved a complex process, meaning that only specialized centres would be able to carry out this therapy. In two recent publications, Steven Rosenberg and colleagues have reported that the generation of these TILs can be simplified, while maintaining their efficacy.

The first study, published in the Journal of Clinical Oncology, addressed the contentious question of whether TILs should be selected to be enriched for $\mathrm{CD}^{+}$cells, or if the unselected population including $\mathrm{CD} 4^{+}$cells is as effective. Patients with metastatic melanoma were randomly assigned to receive either unselected TILs or $\mathrm{CD}^{+}$-enriched TILs after treatment with standard induction therapy.

All of the 69 patients who took part in the trial were treated using the same general protocol at the NCI. Despite this attempt to standardize the protocol there was a substantial variation in the site of tumour procurement, method of TIL expansion, age of TILs at the time of infusion, number of cells infused, percentage of $\mathrm{CD} 4{ }^{+}$cells and the tumour recognition by the TILs.

In the patients who received unselected TILs, 35\% exhibited an objective response, with the two complete responders still in remission at the time of the report. For the patients who received the selected TILs, 20\% achieved an objective response, and of the three complete responses two were ongoing. These results were not significantly different, indicating that the more-simple TIL protocol used in the unselected patients should be the preferred strategy in future clinical trials.

The second study, published in Nature Medicine, focussed on the antigens that are recognized by adoptively transferred autologous TILs. The identification of the antigens associated with a tumour response has been challenging owing to their diversity, but this study used a new screening method to identify mutations in antigens that were expressed on autologous tumours from patients with melanoma.

The method used whole-exome sequencing of tumour-cell DNA followed by the use of MHC binding algorithms to

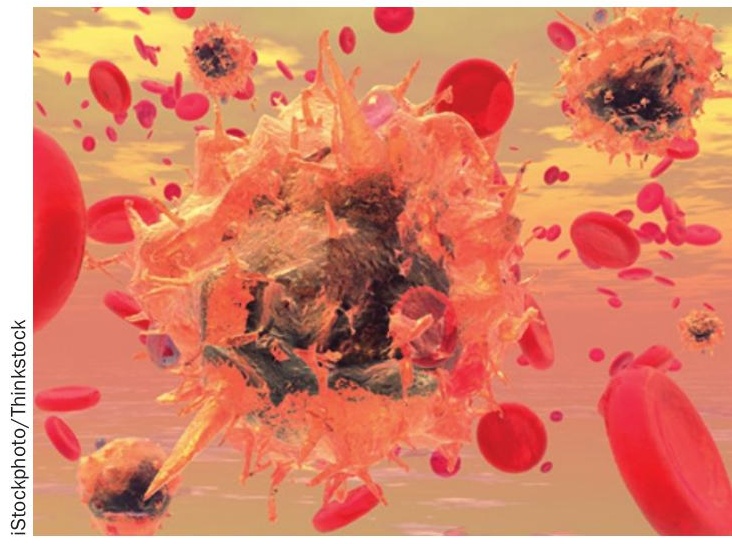

identify mutated epitopes. Some of these epitopes were present in several of the cell lines tested. The authors describe the method as "a relatively simple and rapid genomic approach [that could] provide the means to further explore the relationship between the clinical efficacy of adoptively transferred melanoma-reactive TILs and their ability to recognize mutated T-cell epitopes."

Rebecca Kirk

Original articles Dudley, M. E. et al. Randomized selection
design trial evaluating $\mathrm{CD}^{+}$-enriched versus unselected
tumor-infiltrating lymphocytes for adoptive cell therapy
for patients with melanoma. J. Clin. Oncol. doi:10.1200/
JC0.2012.46.6441 | Robbins, P. F. et al. Mining exomic
sequencing data to identify mutated antigens recognized
by adoptively transferred tumor-reactive T cells. Nat. Med.
doi:10.1038/nm.3161

